\title{
On the exit time from an orthant for badly oriented random walks
}

\author{
Rodolphe Garbit \\ Université d'Angers
}

\begin{abstract}
It was recently proved that the exponential decreasing rate of the probability that a random walk stays in a $d$-dimensional orthant is given by the minimum on this orthant of the Laplace transform of the random walk increments, provided that this minimum exists. In other cases, the random walk is "badly oriented" and the exponential rate may depend on the starting point $x$. We show here that this rate is nevertheless asymptotically equal to the infimum of the Laplace transform, as some selected coordinates of $x$ tend to infinity.
\end{abstract}

\section{Introduction}

\subsection{Context}

This work is a continuation of the paper (Garbit and Raschel (2016)) in which the authors studied the exponential decreasing rate of the probability that a random walk (with some exponential moments) stays in a $d$-dimensional convex cone, and found that this rate is equal to the minimum on the dual cone of the Laplace transform of the random walk increments, provided that this minimum exists. In the present work, we shall restrict our attention to the case where the cone is a $d$ dimensional orthant, and extend the preceding result so as to cover also the cases when there is no minimum. This happens when the random walk is "badly oriented" with respect to the orthant, that is, when the support of the step distribution is included in a half-space whose outward normal is in the orthant.

In order to be more specific, let us introduce some notations. For any fixed probability distribution $\mu$ on $\mathbb{R}^{d}$, let $\mathbb{P}_{\mu}^{x}$ denote the probability measure on $\mathbb{R}^{\infty}$ under which the canonical process $\left(S_{0}, S_{1}, \ldots, S_{n}, \ldots\right)$ is a random walk started at $x$ (meaning that $S_{0}=x$ a.s.) whose independent increments $S_{n+1}-S_{n}$ have distribution $\mu$.

Let $K \subset \mathbb{R}^{d}$ be some convex cone with non-empty interior and let

$$
\tau_{K}=\inf \left\{n \geq 1: S_{n} \notin K\right\}
$$

denote the exit time of the random walk from $K$.

Key words and phrases. Random walk, cones, exit time, Laplace transform.

Received September 2015; accepted August 2016. 
For random walks with no drift, the precise asymptotics

$$
\mathbb{P}_{\mu}^{x}\left(\tau_{K}>n\right)=c \rho^{n} n^{-\alpha}(1+o(1)), \quad n \rightarrow \infty
$$

was derived by Denisov and Wachtel (2015) from the corresponding tail distribution for Brownian motion by using a strong approximation theorem. In that case, $\rho=1$. They also obtained a local limit theorem from which Duraj could derive (Duraj (2014)) the precise asymptotics for random walks with "negative" drift, that is when the global minimum on $\mathbb{R}^{d}$ of the Laplace transform

$$
L_{\mu}(z)=\int_{\mathbb{R}^{d}} e^{\langle z, y\rangle} \mu(d y)
$$

is reached at an interior point of the dual cone

$$
K^{*}=\left\{x \in \mathbb{R}^{d}:\langle x, y\rangle \geq 0, \forall y \in K\right\} .
$$

In that case, he found that $\rho=\min _{\mathbb{R}^{d}} L_{\mu}$. The problem of determining the exponential rate only, but disregarding the position of the global minimum on $\mathbb{R}^{d}$, was solved under general conditions in Garbit and Raschel (2016). In that paper, we found that the right place to look at is the position of the global minimum on the dual cone $K^{*}$ of the Laplace transform. Indeed, the main result in Garbit and Raschel (2016) is that the exponential decreasing rate

$$
\rho_{x}=\liminf _{n \rightarrow \infty} \mathbb{P}_{\mu}^{x}\left(\tau_{K}>n\right)^{1 / n}
$$

is given by the identity

$$
\rho_{x}=\min _{K^{*}} L_{\mu},
$$

for all $x$ far enough from the boundary of $K$, provided that this minimum exists. Note that in this case, there is essentially no dependence in the starting point $x$.

The goal of the present work is to study the case where this minimum does not exist. For technical reasons (that should become clearer when reading the rest of the paper), we shall restrict our attention to the case where $K=Q$ is the positive orthant

$$
Q=\left\{x \in \mathbb{R}^{d}: x_{i} \geq 0, i=1 \cdots d\right\},
$$

where $x_{i}$ denotes the $i$ th coordinate of $x$ with respect to the standard basis $\left(e_{1}, e_{2}, \ldots, e_{d}\right)$. Note that $Q^{*}=Q$. In addition, in order to simplify the exposition, we will assume that the probability distribution $\mu$ has all exponential moments, that is, $L_{\mu}(z)$ is finite for all $z \in \mathbb{R}^{d}$.

For a truly $d$-dimensional distribution $\mu$, that is, a distribution whose support is not included in any linear hyperplane, the condition that $L_{\mu}$ reaches a global minimum on $Q$ is equivalent to the following geometric condition (see Garbit and Raschel (2016) for a proof): 
(H) The support of $\mu$ is not included in any half-space $u^{-}=\left\{x \in \mathbb{R}^{d}:\langle x, u\rangle \leq\right.$ $0\}$ with $u \in Q \backslash\{0\}$.

Random walks with a distribution $\mu$ that fulfills condition $(\mathrm{H})$ are called welloriented whereas the others are called badly oriented. In this second class, the exponential rate $\rho_{x}$ may depend on the starting point $x$, as shown by the following example.

Example 1. Consider the 2-dimensional lattice distribution $\mu$ defined by

$$
\mu(1,-1)=\mu(-1,1)=q, \quad \mu(-1,-1)=p, \quad p+2 q=1, \quad p, q>0 .
$$

The corresponding random walk is badly oriented since the support of $\mu$ is included in $(1,1)^{-}$. Its Laplace transform satisfies the relations

$$
L_{\mu}(i, j)=2 q \cosh (i-j)+p e^{-(i+j)}>2 q,
$$

and

$$
\lim _{i \rightarrow+\infty} L_{\mu}(i, i)=2 q
$$

Therefore, the infimum $2 q$ of $L_{\mu}$ on $Q$ is not a minimum. It is proved in Garbit and Raschel (2016) that, for $x=(i, j) \in \mathbb{N}_{+}^{2}$ with $i+j=2 N$,

$$
\rho_{x}=2 q \cos \left(\frac{\pi}{2 N+2}\right) \text {. }
$$

Thus $\rho_{x}$ depends on $x$, but we nevertheless observe that

$$
\lim _{\|x\| \rightarrow \infty} \rho_{x}=2 q=\inf _{Q} L_{\mu}
$$

The aim of this paper is to give a result that applies to both well and badly oriented random walks for the case of the positive orthant. More precisely, we shall prove that the equality (1) is in fact a particular case of the following equality

$$
\lim \rho_{x}=\inf _{Q} L_{\mu}
$$

where the interpretation of the limit symbol depends on a linear subspace associated with $\mu$ that we call the reduced support of $\mu$.

The central idea in Garbit and Raschel (2016) was to perform a standard Cramér transformation (i.e., an exponential change of measure) based on the point $x_{0}$ where $L_{\mu}$ reaches its minimum on $Q$. The main novelty here is that we provide a way to achieve this transformation when $x_{0}$ is "at infinity". 


\subsection{Reduction}

The assumption $(\mathrm{H})$ was used in Garbit and Raschel (2016) to ensure the existence of a global minimum of $L_{\mu}$ on $Q$. The implication follows easily from the following property of the Laplace transform (see Garbit and Raschel (2016), Lemma 3):

$$
\lim _{t \rightarrow+\infty} L_{\mu}(z+t u)= \begin{cases}+\infty, & \text { if } \mu\left(u^{-}\right)<1, \\ \int_{u^{\perp}} e^{\langle z, y\rangle} \mu(d y), & \text { if } \mu\left(u^{-}\right)=1,\end{cases}
$$

where $u^{\perp}$ denotes the hyperplane orthogonal to $u$. Indeed, for a distribution satisfying assumption $(\mathrm{H})$, the Laplace transform tends to infinity in each direction $u \in Q \cap \mathbb{S}^{d-1}$. Since this function is convex, this implies by a compactness argument that $L_{\mu}$ is coercive in $Q$, and the existence of a global minimum follows.

Suppose now that the random walk is badly oriented, that is, that there exists a direction $u_{1} \in Q \cap \mathbb{S}^{d-1}$ such that $\mu\left(u_{1}^{-}\right)=1$. Then, the "best" way to stay in $Q$ is certainly not to perform a step outside of $u_{1}^{\perp}$. Thus, it seems natural to compare our original random walk with a conditioned version of it, namely the random walk with distribution $v(\cdot)=\mu\left(\cdot \mid u_{1}^{\perp}\right)$. Since this new random walk may still be badly oriented, we shall construct by induction a reduced support $V$ in the following way.

Definition 1. An $r$-tuple $\left(u_{1}, u_{2}, \ldots, u_{r}\right) \in Q^{r}$ is admissible if

(i) the vectors $u_{1}, u_{2}, \ldots, u_{r}$ are linearly independent, and

(ii) they satisfy the following relations:

$$
\left\{\begin{array}{l}
\mu\left(u_{1}^{-}\right)=1, \\
\mu\left(u_{2}^{-} \cap u_{1}^{\perp}\right)=\mu\left(u_{1}^{\perp}\right), \\
\vdots \\
\mu\left(u_{r}^{-} \cap\left(u_{r-1}^{\perp} \cap \cdots \cap u_{1}^{\perp}\right)\right)=\mu\left(u_{r-1}^{\perp} \cap \cdots \cap u_{1}^{\perp}\right) .
\end{array}\right.
$$

An admissible $r$-tuple $\left(u_{1}, u_{2}, \ldots, u_{r}\right)$ is maximal if there is no $u \in Q$ such that $\left(u_{1}, u_{2}, \ldots, u_{r}, u\right)$ be admissible.

If there exists an admissible tuple (i.e., if the random walk is badly oriented), then there exist maximal tuples, and the linear subspace $\left[u_{1}, u_{2}, \ldots, u_{r}\right]$ generated by a maximal tuple does not depend on the specific maximal tuple chosen (Lemma 1).

Definition 2. The orthogonal complement

$$
V=\left[u_{1}, u_{2}, \ldots, u_{r}\right]^{\perp}
$$

of the subspace generated by any maximal tuple is called the reduced support of $\mu$. By convention, if there is no admissible tuple, we set $V=\mathbb{R}^{d}$. 
With the reduced support $V$, we associate the set $I$ of indices $i \in \llbracket 1, d \rrbracket$ such that $e_{i} \in V$, and the cone

$$
V^{+}=\left\{x \in V:\left\langle x, e_{i}\right\rangle \geq 0, \forall i \in I\right\} .
$$

Then (Lemma 2) the infimum on $Q$ of the Laplace transform of $\mu$ is given by the relation

$$
\inf _{Q} L_{\mu}=\inf _{v \in V^{+}} \int_{V} e^{\langle v, y\rangle} \mu(d y)
$$

If $\inf _{Q} L_{\mu}=0$, then

$$
\rho_{x}=\liminf _{n \rightarrow \infty} \mathbb{P}_{\mu}^{x}\left(\tau_{Q}>n\right)^{1 / n}=0
$$

for all $x \in Q$, since $\inf _{Q} L_{\mu}$ is a universal upper bound for $\rho_{x}$ (see equation (5)). Thus, from now on, we will concentrate on the case

$$
\inf _{Q} L_{\mu}>0
$$

Therefore $\mu(V)>0$ and equality (2) can be rewritten as

$$
\inf _{Q} L_{\mu}=\mu(V) \inf _{V^{+}} L_{\mu \mid V}
$$

where $\mu \mid V$ denotes the conditional distribution $\mu \mid V(\cdot)=\mu(\cdot \mid V)$. Now, the "maximality" of $V$ (in the sense of Definition 1) ensures that the conditioned random walk with distribution $\mu \mid V$ is well-oriented with respect to $V^{+}$, that is, that the infimum on $V^{+}$is in fact a minimum (Lemma 3), so that a Cramér transformation can be applied to this conditioned random walk. We interpret this as a Cramér transformation at infinity.

\subsection{Main result}

In what follows, $\mu$ is any probability distribution on $\mathbb{R}^{d}$ with all exponential moments. We denote by $V$ the reduced support of $\mu$ (see Definitions 1 and 2), and define

$$
I=\left\{i \in \llbracket 1, d \rrbracket: e_{i} \in V\right\}, \quad I^{\perp}=\left\{i \in \llbracket 1, d \rrbracket: e_{i} \in V^{\perp}\right\},
$$

and, for $x$ in $Q$,

$$
d(x)=\min _{i \notin I \cup I^{\perp}} x_{i}
$$

with the convention that $d(x)=\infty$ when $I \cup I^{\perp}=\llbracket 1, d \rrbracket$. Finally, we set

$$
Q_{\delta}=Q+\delta(1,1, \ldots, 1)=\left\{x \in \mathbb{R}^{d}: x_{i} \geq \delta, \forall i \in \llbracket 1, d \rrbracket\right\} .
$$

We are now in position to state our main result. 
Theorem 1. There exists $\delta \geq 0$ such that

$$
\lim _{\substack{d(x) \rightarrow \infty \\ x \in Q_{\delta}}} \rho_{x}=\inf _{Q} L_{\mu}
$$

Let us illustrate this theorem with some basic examples.

Example 2. If $\mu$ satisfies assumption $(\mathrm{H})$, then $V=\mathbb{R}^{d}, I=\llbracket 1, d \rrbracket, V^{+}=Q$, $I^{\perp}=\varnothing$, and $d(x)=\infty$. Thus, we recover the non-asymptotic theorem of Garbit and Raschel (2016): There exists $\delta \geq 0$ such that

$$
\rho_{x}=\inf _{Q} L_{\mu},
$$

for all $x \in Q_{\delta}$.

Example 3. Consider the 2-dimensional lattice distribution $\mu$ defined by

$$
\begin{aligned}
\mu(-1,0) & =\alpha, & \mu(0,1)=\beta, & \mu(0,-1)=\gamma, \\
\alpha+\beta+\gamma & =1, & \alpha, \beta, \gamma>0 . &
\end{aligned}
$$

The associated random walk is badly oriented since $\mu\left(e_{1}^{-}\right)=1$. Now, $\left(e_{1}\right)$ is maximal since $\beta, \gamma>0$, therefore $V=e_{1}^{\perp}, I=\{2\}, V^{+}=\{(0, j): j \geq 0\}, I^{\perp}=\{1\}$ and $d(x)=\infty$, meaning that

$$
\rho_{x}=\inf _{Q} L_{\mu},
$$

for all $x \in Q_{\delta}$, for some constant $\delta \geq 0$. Let us compute the value of $\rho_{x}$. The Laplace transform of $\mu$ is given by

$$
L_{\mu}(i, j)=\alpha e^{-i}+\beta e^{j}+\gamma e^{-j} .
$$

Minimizing first on $i \geq 0$ leads to the relation

$$
\inf _{Q} L_{\mu}=\inf _{j \geq 0}\left(\beta e^{j}+\gamma e^{-j}\right)=(\beta+\gamma) \inf _{j \geq 0}\left(\beta^{\prime} e^{j}+\gamma^{\prime} e^{-j}\right),
$$

where $\beta^{\prime}=\beta /(\beta+\gamma)$ and $\gamma^{\prime}=\gamma /(\beta+\gamma)$ sum up to 1 . Notice that the above formula corresponds exactly to equality (3) with $V=e_{1}^{\perp}$. An easy computation shows that

$$
\inf _{j \geq 0}\left(\beta^{\prime} e^{j}+\gamma^{\prime} e^{-j}\right)= \begin{cases}2 \sqrt{\beta^{\prime} \gamma^{\prime}}, & \text { if } \gamma^{\prime} \geq \beta^{\prime} \\ 1, & \text { else. }\end{cases}
$$

Hence

$$
\rho_{x}=\inf _{Q} L_{\mu}= \begin{cases}2 \sqrt{\beta \gamma}, & \text { if } \gamma \geq \beta \\ \beta+\gamma, & \text { else. }\end{cases}
$$


Example 4. Consider the 2-dimensional lattice distribution $\mu$ defined by

$$
\begin{aligned}
& \mu(-1,-1)=\alpha, \quad \mu(-1,1)=\beta, \quad \mu(1,-1)=\gamma, \\
& \alpha+\beta+\gamma=1, \quad \alpha, \beta, \gamma>0 .
\end{aligned}
$$

The associated random walk is badly oriented since the support of $\mu$ is included in $(1,1)^{-}$. Here, $V=(1,1)^{\perp}, I=I^{\perp}=\varnothing, V^{+}=V$ and $d(x)=\min \left\{x_{1}, x_{2}\right\}$. Therefore, we obtain

$$
\lim _{x_{1}, x_{2} \rightarrow \infty} \rho_{x}=\inf _{Q} L_{\mu}=2 \sqrt{\beta \gamma},
$$

as the reader may check.

The rest of the paper is organized as follows: In Section 2, we present the proof of Theorem 1. The construction of the reduced support enables us to perform a Cramér's transformation at infinity, and then compare our initial exponential decreasing rate with that of a conditioned random walk whose distribution is more "favorable". The exponential rate of this conditioned random walk is then analysed in Section 3. The Appendix at the end of the paper provides some material on polyhedra that is needed in a technical lemma and for which no reference were found.

\section{Proof of Theorem 1}

The first subsection recalls the strategy used in Garbit and Raschel (2016) in order to obtain the exponential decreasing rate in the case where the random walk is welloriented. Though not necessary, its reading is recommended in order to become acquainted with the basic ideas of the proof of Theorem 1. We will then present the construction and the main properties of the reduced support, and finally use it to perform our Cramér's transformation at infinity in order to conclude the proof of Theorem 1.

\subsection{Sketch of proof for well-oriented random walks}

It is easy to see that $\rho_{x}=1$ when the drift vector $m=\mathbb{E}(\mu)$ belongs to the orthant $Q$. In other cases, the basic idea is to carry out an exponential change of measure in order to transform the drift vector $m$ into a new one, $m_{0}$, that belongs to $Q$. To do this, fix any $x_{0} \in \mathbb{R}^{d}$ and define

$$
\mu_{0}(d y)=\frac{e^{\left\langle x_{0}, y\right\rangle}}{L_{\mu}\left(x_{0}\right)} \mu(d y) .
$$

Clearly, $\mu_{0}$ is a probability distribution with Laplace transform

$$
L_{\mu_{0}}(z)=\frac{L_{\mu}\left(z+x_{0}\right)}{L_{\mu}\left(x_{0}\right)} .
$$


Furthermore, it is well known that the expectation of a probability distribution is equal to the gradient of its Laplace transform evaluated at 0 . Thus,

$$
m_{0}=\mathbb{E}\left(\mu_{0}\right)=\nabla L_{\mu}\left(x_{0}\right) / L_{\mu}\left(x_{0}\right) .
$$

If $L_{\mu}$ reaches a global minimum on $Q$ at $x_{0}$, then its partial derivatives are nonnegative at $x_{0}$ and, therefore, the drift $m_{0}$ belongs to $Q$. So, the distribution $\mu_{0}$ is "nice" in the sense that the value of the exponential rate for a random walk with that distribution is known. The link between the distribution of our canonical random walk under $\mathbb{P}_{\mu}^{x}$ and $\mathbb{P}_{\mu_{0}}^{x}$ is expressed via Cramér's formula (see Garbit and Raschel (2016), Lemma 6, for example) which leads in our case to the relation

$$
\mathbb{P}_{\mu}^{x}\left(\tau_{Q}>n\right)=L_{\mu}\left(x_{0}\right)^{n} e^{\left\langle x_{0}, x\right\rangle} \mathbb{E}_{\mu_{0}}^{x}\left(e^{-\left\langle x_{0}, S_{n}\right\rangle}, \tau_{Q}>n\right) .
$$

From this, we already notice that for all $x_{0} \in Q$ (being a minimum point or not),

$$
\limsup _{n \rightarrow \infty} \mathbb{P}_{\mu}^{x}\left(\tau_{Q}>n\right)^{1 / n} \leq L_{\mu}\left(x_{0}\right),
$$

since $\left\langle x_{0}, S_{n}\right\rangle \geq 0$ as soon as $S_{n} \in Q$. Thus, the upper bound

$$
\limsup _{n \rightarrow \infty} \mathbb{P}_{\mu}^{x}\left(\tau_{Q}>n\right)^{1 / n} \leq \inf _{Q} L_{\mu}
$$

holds for any probability distribution $\mu$ (with all exponential moments).

In order to obtain the corresponding lower bound in the case where $L_{\mu}$ reaches a global minimum on $Q$ at $x_{0}$, we use formula (4) with our nice distribution $\mu_{0}$. Now, the problem is to bound from below the $\exp \left(-\left\langle x_{0}, S_{n}\right\rangle\right)$ term, which could become very small since $\left(S_{n}\right)$ has a drift $m_{0} \in Q$. But, in fact, the growth of $\left\langle x_{0}, S_{n}\right\rangle$ can be controlled thanks to the following observation: Let $K$ be the set of indices $i$ such that $x_{0}^{(i)}>0$. Since $x_{0}$ belongs to $Q$, the other coordinates are equal to zero, and

$$
\left\langle x_{0}, S_{n}\right\rangle=\sum_{i \in K} x_{0}^{(i)} S_{n}^{(i)} .
$$

Furthermore, if $x_{0}^{(i)}>0$ for some index $i$, then 0 is a local minimum of the partial function $t \in\left[-x_{0}^{(i)},+\infty\right) \mapsto L_{\mu}\left(x_{0}+t e_{i}\right)$ and therefore $m_{0}^{(i)}=0$, because it is proportional to $\partial_{x_{i}} L_{\mu}\left(x_{0}\right)=0$. So, the coordinates of the random walk that we need to control have zero mean. Since $\sqrt{n}$ is a natural bound for the norm of a square integrable centered random walk, one can expect that adding the constraint

$$
\max _{i \in K}\left|S_{n}^{(i)}\right| \leq \sqrt{n}
$$

will not decrease too much the probability on the right-hand side of equation (4). As proved in Garbit and Raschel (2016) this happens to be true: For any distribution $\mu$ satisfying assumption $(\mathrm{H})$,

$$
\liminf _{n \rightarrow \infty} \mathbb{P}_{\mu_{0}}^{x}\left(\max _{i \in K}\left|S_{n}^{(i)}\right| \leq \sqrt{n}, \tau_{Q}>n\right)^{1 / n}=1
$$


Under the constraint (6), the exponential term $\exp \left(-\left\langle x_{0}, S_{n}\right\rangle\right)$ in (4) is bounded from below by $\exp \left(-\left\|x_{0}\right\| \sqrt{n}\right)$, a term that disappears in the $n$th root limit. Thus, we obtain the lower bound

$$
\rho_{x} \geq L_{\mu}\left(x_{0}\right) \liminf _{n \rightarrow \infty} \mathbb{P}_{\mu_{0}}^{x}\left(\max _{i \in K}\left|S_{n}^{(i)}\right| \leq \sqrt{n}, \tau_{Q}>n\right)^{1 / n}=L_{\mu}\left(x_{0}\right),
$$

and this concludes the analysis.

\subsection{Construction and properties of the reduced support}

In this section, we prove the uniqueness of the reduced support of $\mu$ (Definitions 1 and 2) and establish some of its properties. When $\mu$ satisfy assumption (H), the reduced support $V$ is equal to $\mathbb{R}^{d}$ - by definition-and all properties listed below are trivial, as the reader may check. Therefore, in what follows, we assume the existence of a direction $u_{1} \in Q \cap \mathbb{S}^{d-1}$ such that $\mu\left(u_{1}^{-}\right)=1$. Thus, there exists at least an admissible tuple, namely $\left(u_{1}\right)$, and the existence of a maximal tuple follows immediately.

First, we prove uniqueness ${ }^{1}$ of the linear space generated by maximal admissible tuples.

Lemma 1. Any two maximal admissible tuples generate the same linear space.

Proof. Let $\left(u_{1}, u_{2}, \ldots, u_{r}\right)$ and $\left(v_{1}, v_{2}, \ldots, v_{s}\right)$ be two maximal admissible tuples and let

$$
A=\left[u_{1}, u_{2}, \ldots, u_{r}\right] \text { and } B=\left[v_{1}, v_{2}, \ldots, v_{s}\right]
$$

denote the linear spaces generated by this two tuples, respectively. Since $\left(u_{1}\right.$, $\left.u_{2}, \ldots, u_{r}\right)$ is maximal, $\left(u_{1}, u_{2}, \ldots, u_{r}, v_{1}\right)$ is not admissible. On the other hand, $\mu\left(v_{1}^{-}\right)=1$ so that

$$
\mu\left(v_{1}^{-} \cap A^{\perp}\right)=\mu\left(A^{\perp}\right),
$$

and the tuple $\left(u_{1}, u_{2}, \ldots, u_{r}, v_{1}\right)$ satisfies condition (ii) of Definition 1 . Thus, the reason why this tuple fails to be admissible is that condition (i) is not satisfied, that is, $v_{1}$ belongs to $A$. Now, suppose that $v_{1}, v_{2}, \ldots, v_{k-1}$ all belong to $A$ for some $k \in \llbracket 2, s \rrbracket$, and denote $B_{k}=\left[v_{1}, v_{2}, \ldots, v_{k-1}\right]$ the linear space they generate. By hypothesis, we have

$$
A^{\perp} \subset B_{k}^{\perp} \quad \text { and } \quad \mu\left(v_{k}^{-} \cap B_{k}^{\perp}\right)=\mu\left(B_{k}^{\perp}\right) .
$$

This implies $\mu\left(v_{k}^{-} \cap A^{\perp}\right)=\mu\left(A^{\perp}\right)$ and therefore $v_{k}$ must belong to $A$, otherwise $\left(u_{1}, u_{2}, \ldots, u_{r}, v_{k}\right)$ would be admissible. By induction, we obtain the inclusion $B \subset A$, and the equality $A=B$ follows by interchanging the role of $A$ and $B$.

\footnotetext{
${ }^{1}$ This property will not be used, but it is a very natural question since the conclusion of Theorem 1 depends on this subspace.
} 
Thanks to Lemma 1 we can define the reduced support $V$ of $\mu$ as the orthogonal complement of the linear space generated by any maximal admissible tuple (Definition 2). In what follows, we fix a maximal admissible tuple $\left(u_{1}, u_{2}, \ldots, u_{r}\right)$ and set

$$
V=\left[u_{1}, u_{2}, \ldots, u_{r}\right]^{\perp} \text { and } V^{+}=V \cap\left(\bigcup_{h \in V^{\perp}} Q-h\right) .
$$

The reduced support $V$ and the cone $V^{+}$play a fundamental role in our analysis. First of all, they provide a useful expression for the infimum of the Laplace transform.

Lemma 2. The following equality holds:

$$
\inf _{Q} L_{\mu}=\inf _{v \in V^{+}} \int_{V} e^{\langle v, y\rangle} \mu(d y) \text {. }
$$

Proof. It follows from the orthogonal decomposition $\mathbb{R}^{d}=V \oplus V^{\perp}$ that

$$
\begin{aligned}
\inf \left\{L_{\mu}(x): x \in Q\right\} & =\inf \left\{L_{\mu}(v+h):(v, h) \in V \times V^{\perp}, v+h \in Q\right\} \\
& =\inf _{v \in V^{+}} \inf _{h \in V^{\perp} \cap(Q-v)} L_{\mu}(v+h) .
\end{aligned}
$$

Therefore, we have to prove that for all $v \in V^{+}$

$$
\inf _{h \in V^{\perp} \cap(Q-v)} L_{\mu}(v+h)=\int_{V} e^{\langle v, y\rangle} \mu(d y) .
$$

Let $v \in V^{+}$. First, for all $h \in V^{\perp}$, we notice that

$$
L_{\mu}(v+h) \geq \int_{V} e^{\langle v+h, y\rangle} \mu(d y)=\int_{V} e^{\langle v, y\rangle} \mu(d y),
$$

since $\langle h, y\rangle=0$ for all $y \in V$. Now, pick $h \in V^{\perp} \cap(Q-v)$ (such a $h$ exists since $v \in V^{+}$), and let $\lambda_{1}, \lambda_{2}, \ldots, \lambda_{r}$ denote its coordinates with respect to the basis $\left(u_{1}, u_{2}, \ldots, u_{r}\right)$ of $V^{\perp}$. It is clear that $h=\sum_{k=1}^{r} \lambda_{k} u_{k}$ will still belong to $V^{\perp} \cap(Q-v)$ if one increases the value of any $\lambda_{i}$. Hence, the equality (7) will follow from

$$
\lim _{\lambda_{r} \rightarrow \infty} \cdots \lim _{\lambda_{2} \rightarrow \infty} \lim _{\lambda_{1} \rightarrow \infty} L_{\mu}\left(v+\sum_{k=1}^{r} \lambda_{r} u_{r}\right)=\int_{V} e^{\langle v, y\rangle} \mu(d y) .
$$

Since $\left(u_{1}, u_{2}, \ldots, u_{r}\right)$ is admissible, $\mu\left(u_{1}^{-}\right)=1$ and consequently

$$
\begin{aligned}
L_{\mu}\left(v+\sum_{k=1}^{r} \lambda_{r} u_{r}\right)= & \int_{u_{1}^{\perp}} e^{\langle v, y\rangle} e^{\sum_{k=2}^{r} \lambda_{k}\left\langle u_{k}, y\right\rangle} \mu(d y) \\
& +\int_{\left\{\left\langle u_{1}, y\right\rangle<0\right\}} e^{\langle v, y\rangle} e^{\sum_{k=1}^{r} \lambda_{k}\left\langle u_{k}, y\right\rangle} \mu(d y) .
\end{aligned}
$$


By the dominated convergence theorem, the second integral on the right-hand side of the above equation goes to zero as $\lambda_{1}$ goes to infinity. Hence

$$
\lim _{\lambda_{1} \rightarrow \infty} L_{\mu}\left(v+\sum_{k=1}^{r} \lambda_{r} u_{r}\right)=\int_{u_{1}^{\perp}} e^{\langle v, y\rangle} e^{\sum_{k=2}^{r} \lambda_{k}\left\langle u_{k}, y\right\rangle} \mu(d y) .
$$

Now, by hypothesis, we have $\mu\left(u_{2}^{-} \cap u_{1}^{\perp}\right)=\mu\left(u_{1}^{\perp}\right)$, so that the same argument as above leads to

$$
\lim _{\lambda_{2} \rightarrow \infty} \lim _{\lambda_{1} \rightarrow \infty} L_{\mu}\left(v+\sum_{k=1}^{r} \lambda_{r} u_{r}\right)=\int_{u_{1}^{\perp} \cap u_{2}^{\perp}} e^{\langle v, y\rangle} e^{\sum_{k=3}^{r} \lambda_{k}\left\langle u_{k}, y\right\rangle} \mu(d y) .
$$

The equality in (8) is obtained by using repeatedly the same argument.

Lemma 3. Assume $\inf _{Q} L_{\mu}>0$. Then:

1. $\mu(V)>0$.

2. For all $v \in V^{+} \cap \mathbb{S}^{d-1}, \mu\left(v^{-} \mid V\right)<1$.

3. The Laplace transform $L_{\mu \mid V}$ has a global minimum on $V^{+}$.

4. $\inf _{Q} L_{\mu}=\mu(V) \min _{V^{+}} L_{\mu \mid V}$.

Proof. The formula in Lemma 2 shows that $\inf _{Q} L_{\mu}=0$ as soon as $\mu(V)=0$. The first item follows by contraposition.

Let $v \in V^{+} \cap \mathbb{S}^{d-1}$. By definition of $V^{+}$there exists $h \in V^{\perp}$ such that $u=$ $v+h \in Q$. For any $y \in V$, we have $\langle v, y\rangle=\langle u, y\rangle$ and consequently

$$
v^{-} \cap V=u^{-} \cap V
$$

So, it suffices to show that $\mu\left(u^{-} \mid V\right)<1$ and the second assertion of the lemma will follow. By definition, $u=v+h$ (with $v \in V, v \neq 0$ and $h \in V^{\perp}$ ) is linearly independent of $V^{\perp}=\left[u_{1}, u_{2}, \ldots, u_{r}\right]$ and belongs to $Q$. But by maximality, the tuple $\left(u_{1}, u_{2}, \ldots, u_{r}, u\right)$ is not admissible. Thus, we must have $\mu\left(u^{-} \cap V\right)<\mu(V)$. This proves the second assertion of the lemma.

The third assertion follows from the second one since $V^{+}$is a closed cone (see Garbit and Raschel (2016), Lemma 4-note that the hypothesis (H1) is not used for the part of the lemma that we need here).

Finally, the last item is just a reformulation of the formula in Lemma 2.

We shall now give a very simple description of the cone $V^{+}$associated with the reduced support $V$. To this end, we define

$$
I=\left\{i \in \llbracket 1, d \rrbracket: e_{i} \in V\right\} .
$$

Lemma 4. The cone $V^{+}$has the following expression:

$$
V^{+}=\left\{x \in V:\left\langle x, e_{i}\right\rangle \geq 0, \forall i \in I\right\} .
$$


Proof. We first note that $x \in \bigcup_{h \in V^{\perp}} Q-h$ if and only if there exists $h \in V^{\perp}$ such that $\left\langle x+h, e_{i}\right\rangle \geq 0$ for all $i$. But, for $i \in I,\left\langle x+h, e_{i}\right\rangle=\left\langle x, e_{i}\right\rangle$ since $e_{i} \in V$ and $h \in V^{\perp}$. Hence, the condition splits into:

1. $\left\langle x, e_{i}\right\rangle \geq 0$ for all $i \in I$, and

2. there exists $h \in V^{\perp}$ such that $\left\langle x+h, e_{i}\right\rangle \geq 0$ for all $i \notin I$.

Therefore, it remains to prove that the last condition holds for all $x$. To this end, recall that $V^{\perp}=\left[u_{1}, u_{2}, \ldots, u_{r}\right]$ with $u_{k} \in Q$, and set $u_{0}=\sum_{k=1}^{r} u_{k}$. Since $\left\langle u_{0}, e_{i}\right\rangle=\sum_{k=1}^{r}\left\langle u_{k}, e_{i}\right\rangle$ and $\left\langle u_{k}, e_{i}\right\rangle \geq 0$ for all $k$, we see that $\left\langle u_{0}, e_{i}\right\rangle \geq 0$ and equality occurs if and only if $\left\langle u_{k}, e_{i}\right\rangle=0$ for all $k$, which means exactly that $e_{i} \in\left[u_{1}, u_{2}, \ldots, u_{r}\right]^{\perp}=V$. Thus, by definition of the set $I$, we have $\left\langle u_{0}, e_{i}\right\rangle>0$ for all $i \notin I$. For any fixed $x$, this property allows to find $\lambda>0$ such that $\left\langle x, e_{i}\right\rangle+\lambda\left\langle u_{0}, e_{i}\right\rangle \geq 0$ for all $i \notin I$, so that condition 2 holds with $h=\lambda u_{0}$. This proves the lemma.

\subsection{Comparison with the conditioned random walk with increments restricted to the reduced support}

As mentioned earlier (see (5)), the infimum of $L_{\mu}$ on $Q$ is always an upper bound for the exponential decreasing rate

$$
\rho_{x}=\liminf _{n \rightarrow \infty} \mathbb{P}_{\mu}^{x}\left(\tau_{Q}>n\right)^{1 / n} .
$$

Therefore, our task is to show that it is also a lower bound for $\rho_{x}$ (at least as $x \rightarrow \infty$ in the sense of Theorem 1).

Let $V$ be the reduced support of $\mu$. From now on, we assume that $\inf _{Q} L_{\mu}>0$, so that $\mu(V)>0$ (Lemma 3). Remember that we have introduced the reduced support with the idea that the best way to stay in $Q$ was to never perform any step outside of $V$. Hence, denoting $\xi_{1}, \xi_{2}, \ldots, \xi_{n}$ the increments of the random walk, it is natural to use the lower bound

$$
\begin{aligned}
\mathbb{P}_{\mu}^{x}\left(\tau_{Q}>n\right)^{1 / n} & \geq \mathbb{P}_{\mu}^{x}\left(\tau_{Q}>n, \xi_{1}, \xi_{2}, \ldots, \xi_{n} \in V\right)^{1 / n} \\
& =\mu(V) \mathbb{P}_{\mu \mid V}^{x}\left(\tau_{Q}>n\right)^{1 / n} .
\end{aligned}
$$

A look at the last formula of Lemma 3,

$$
\inf _{Q} L_{\mu}=\mu(V) \min _{V^{+}} L_{\mu \mid V}
$$

then explains our strategy: Theorem 1 will follow from a comparison between

$$
\liminf _{n \rightarrow \infty} \mathbb{P}_{\mu \mid V}^{x}\left(\tau_{Q}>n\right)^{1 / n} \quad \text { and } \min _{V^{+}} L_{\mu \mid V}
$$

To simplify notations, set $v=\mu \mid V$. For all $x$ in $Q$, write $x=v+w$ the orthogonal decomposition with respect to $V$ and $V^{\perp}$. Then

$$
\mathbb{P}_{v}^{x}\left(\tau_{Q}>n\right)=\mathbb{P}_{v}^{v}\left(S_{1}, S_{2}, \ldots, S_{n} \in Q-w\right) .
$$


Under $\mathbb{P}_{v}^{v}$, the random walk $S_{1}, S_{2}, \ldots, S_{n}$ almost surely belongs to $V$ (since $v \in V$, and $v(V)=1$ ). Thus, we have to focus our attention on the geometry of $(Q-w) \cap V$.

First, recall the definitions of $I, I^{\perp}$ and $d(x)$ at the beginning of Section 1.3, and notice that $\|a-x\| \leq d(x)$ implies that $a_{i} \geq 0$ for all $i \notin I \cup I^{\perp}$.

Let $V_{1}=\left[e_{i}, i \in I\right]$ and write $V=V_{1} \oplus V_{2}$ the orthogonal decomposition of $V$. Define the positive orthant of $V_{1}$ as

$$
V_{1}^{+}=\left\{y \in V_{1}:\left\langle y, e_{i}\right\rangle \geq 0, \forall i \in I\right\}
$$

and notice that Lemma 4 asserts that

$$
V^{+}=V_{1}^{+} \oplus V_{2} \text {. }
$$

For any $y \in V$, let $y^{(1)}$ and $y^{(2)}$ be the projections of $y$ onto $V_{1}$ and $V_{2}$, respectively.

Lemma 5. For all $x=v+w \in Q$,

$$
\left\{y \in V: y^{(1)} \in V_{1}^{+} \text {and }\left\|y^{(2)}-v^{(2)}\right\| \leq d(x)\right\} \subset(Q-w) \cap V .
$$

Proof. Let $y \in V$ be such that $y^{(1)} \in V_{1}^{+}$and $\left\|y^{(2)}-v^{(2)}\right\| \leq d(x)$. We have to show that $y+w$ belongs to $Q$.

First of all, for any $i \in I^{\perp}$, we have $e_{i} \in V^{\perp}$ so that

$$
\left\langle y+w, e_{i}\right\rangle=\left\langle v+w, e_{i}\right\rangle=x_{i} \geq 0 .
$$

Similarly, since $\left\langle y+w, e_{i}\right\rangle=\left\langle y^{(1)}, e_{i}\right\rangle$ for all $i \in I$, the condition $y^{(1)} \in V_{1}^{+}$ rewrites $\left\langle y+w, e_{i}\right\rangle \geq 0$ for all $i \in I$.

It remains to check that the conclusion also holds when $i \notin I \cup I^{\perp}$. To this end, we notice that

$$
\left\|\left(v^{(1)}+y^{(2)}+w\right)-x\right\|=\left\|y^{(2)}-v^{(2)}\right\| \leq d(x) .
$$

Therefore, for all $i \notin I \cup I^{\perp}$,

$$
\left\langle v^{(1)}+y^{(2)}+w, e_{i}\right\rangle \geq 0 .
$$

But for those indices $i$, we have $e_{i} \perp V_{1}$ and consequently

$$
\left\langle y+w, e_{i}\right\rangle=\left\langle v^{(1)}+y^{(2)}+w, e_{i}\right\rangle \geq 0 .
$$

This concludes the proof of the lemma.

This lemma provides the convenient lower bound:

$$
\mathbb{P}_{v}^{x}\left(\tau_{Q}>n\right) \geq \mathbb{P}_{v}^{v}\left(\tau_{V^{+}}>n, \max _{k \leq n}\left\|S_{k}^{(2)}-v^{(2)}\right\| \leq d(x)\right) .
$$

We now analyse this lower bound with the help of Cramér's transformation. We know by Lemma 3 that there exists $v_{0} \in V^{+}$such that

$$
\lambda:=L_{v}\left(v_{0}\right)=\min _{V^{+}} L_{v}>0 .
$$


Let $v_{0}$ be the probability measure on $V$ defined by

$$
\lambda v_{0}(d y)=e^{\left\langle v_{0}, y\right\rangle} v(d y) .
$$

Thanks to Cramér's formula (see Garbit and Raschel (2016), Lemma 6), the lower bound in equation (11) can be written as

$$
\lambda^{n} \mathbb{E}_{v_{0}}^{v}\left(e^{-\left\langle v_{0}, S_{n}-v\right\rangle}, \tau_{V^{+}}>n, \max _{k \leq n}\left\|S_{k}^{(2)}-v^{(2)}\right\| \leq d(x)\right) .
$$

Since $v_{0} \in V^{+}$, we have $\left\langle v_{0}, e_{i}\right\rangle \geq 0$ for all $i \in I$. Define

$$
K=\left\{i \in I:\left\langle v_{0}, e_{i}\right\rangle>0\right\} .
$$

Then $\left\langle v_{0}, e_{i}\right\rangle=0$ for all $i \in I \backslash K$, so that

$$
\begin{aligned}
\left|\left\langle v_{0}, S_{n}-v\right\rangle\right| & =\left|\sum_{i \in K}\left\langle v_{0}, e_{i}\right\rangle\left\langle S_{n}^{(1)}-v^{(1)}, e_{i}\right\rangle+\left\langle v_{0}, S_{n}^{(2)}-v^{(2)}\right\rangle\right| \\
& \leq\left(\sum_{i \in K}\left\langle v_{0}, e_{i}\right\rangle+\left\|v_{0}\right\|\right) d(x),
\end{aligned}
$$

as soon as $\left|\left\langle S_{n}^{(1)}-v^{(1)}, e_{i}\right\rangle\right| \leq d(x)$ for all $i \in K$ and $\left\|S_{n}^{(2)}-v^{(2)}\right\| \leq d(x)$. Under this additional constraint, the term $\exp \left(-\left\langle v_{0}, S_{n}\right\rangle\right)$ inside the expectation in (12) is bounded from below by some positive constant that will disappear in the $n$th root limit.

Therefore, using the notation

$$
\widetilde{\rho}_{x}=\liminf _{n \rightarrow \infty} \mathbb{P}_{v}^{x}\left(\tau_{Q}>n\right)^{1 / n},
$$

we have

$$
\tilde{\rho}_{x} \geq \lambda \liminf _{n \rightarrow \infty} \mathbb{P}_{v_{0}}^{v}\left(\tau_{V^{+}}>n, \max _{k \leq n} \chi\left(S_{k}-v\right) \leq d(x)\right)^{1 / n}
$$

where

$$
\chi\left(S_{k}-v\right)=\max \left\{\max _{i \in K}\left|\left\langle S_{k}^{(1)}-v^{(1)}, e_{i}\right\rangle\right|,\left\|S_{k}^{(2)}-v^{(2)}\right\|\right\} .
$$

The behavior of this last limit is analysed in Proposition 1, which will be stated and proved in the third section of the paper. Indeed, the space $V$ where the probability distribution $v$ lives has the cartesian product structure $V=V_{1} \oplus V_{2}$ and the cone writes $V^{+}=V_{1}^{+} \oplus V_{2}$, where $V_{1}^{+}$is the positive orthant of $V_{1}$. Furthermore, Lemma 3 asserts that $v=\mu \mid V$ satisfies assumption $\left(\mathrm{H}^{\prime}\right)$ of Proposition 1 with respect to $V^{+}$; so does $\nu_{0}$ since $v$ and $\nu_{0}$ are absolutely continuous with respect to each other. Thus, it remains to take a look at its expectation $m_{0}$ which is given by

$$
m_{0}=\nabla L_{v_{0}}(0)=\lambda^{-1} \nabla L_{v}\left(v_{0}\right) .
$$

Let us write $m_{0}=m_{0}^{(1)}+m_{0}^{(2)}$ with $m_{0}^{(1)} \in V_{1}$ and $m_{0}^{(2)} \in V_{2}$. Since $v_{0}$ is a global minimum point of $L_{v}$ on $V^{+}=V_{1}^{+} \oplus V_{2}$, it is easily seen that: 
- $m_{0}^{(1)} \in V_{1}^{+}$,

- $\left\langle m_{0}^{(1)}, e_{i}\right\rangle=0$ for all $i \in K$, and

- $m_{0}^{(2)}=0$.

Indeed, for all $i \in I$, we have $v_{i}=\left\langle v_{0}, e_{i}\right\rangle \geq 0$, and the half-line $\left\{v_{0}+t e_{i}: t \geq\right.$ $\left.-v_{i}\right\}$ is included in $V^{+}$. Therefore, the function

$$
t \in\left[-v_{i}, \infty\right) \mapsto f_{i}(t)=L_{v}\left(v_{0}+t e_{i}\right)
$$

reaches its minimum at $t=0$. This implies that $\left\langle m_{0}^{(1)}, e_{i}\right\rangle=\lambda^{-1} f_{i}^{\prime}(0) \geq 0$ with equality if $v_{i}>0$, i.e. if $i \in K$. On the other hand, if we take any $h \in V_{2}$ then the whole line $\left\{v_{0}+t h: t \in \mathbb{R}\right\}$ is included in $V^{+}$, hence the function $t \in \mathbb{R} \mapsto$ $L_{v}\left(v_{0}+t h\right)$ reaches a local minimum at $t=0$. By consequence, its derivative $\left\langle\nabla L_{v}(0), h\right\rangle=\lambda\left\langle m_{0}, h\right\rangle$ at $t=0$ is always equal to 0 . Thus, $m_{0}^{(2)}=0$.

Thanks to those properties of $v_{0}$ and its expectation $m_{0}$, we can apply Proposition 1 which ensures the existence of some $\delta \geq 0$ such that

$$
\lim _{R \rightarrow \infty} \liminf _{n \rightarrow \infty} \mathbb{P}_{v_{0}}^{v}\left(\tau_{V^{+}}>n, \max _{k \leq n} \chi\left(S_{k}-v\right) \leq R\right)^{1 / n}=1,
$$

for all $v \in V_{\delta}^{+}=\left\{v \in V: v_{i} \geq \delta, \forall i \in I\right\}$. Notice that the probability under consideration reaches its minimum on $V_{\delta}^{+}$when $v$ is the "corner" point $v_{*}=\delta \sum_{i \in I} e_{i}$ (this follows by inclusion of events). Hence, it follows from (13) that for any $x=v+w \in Q$ with $v \in V_{\delta}^{+}$,

$$
\tilde{\rho}_{x} \geq \lambda \liminf _{n \rightarrow \infty} \mathbb{P}_{\nu_{0}^{*}}^{v_{*}}\left(\tau_{V^{+}}>n, \max _{k \leq n} \chi\left(S_{k}-v_{*}\right) \leq d(x)\right)^{1 / n} .
$$

Since $d(x)$ is now disconnected from the starting point $v_{*}$ we can let $d(x) \rightarrow \infty$, thus proving that

$$
\lim _{\substack{d(x) \rightarrow \infty \\ x \in Q_{\delta}}} \widetilde{\rho}_{x} \geq \lambda=\min _{V^{+}} L_{\mu \mid V}
$$

Theorem 1 then follows from the combination of this inequality with (9) and (10).

\section{The favorable case}

In this section, we consider a square integrable random walk $\left(S_{n}\right)$ in $\mathbb{R}^{d}=\mathbb{R}^{p} \times \mathbb{R}^{q}$ with distribution $\mu$, mean $m$ and variance-covariance matrix $\Gamma$. For all $x \in \mathbb{R}^{d}$, we denote by $x_{i}, i=1 \cdots d$, its coordinates in the standard basis, and $x^{(1)} \in \mathbb{R}^{p}$ and $x^{(2)} \in \mathbb{R}^{q}$ its "coordinates" with respect to the cartesian product $\mathbb{R}^{p} \times \mathbb{R}^{q}$. Let $Q$ denote the positive orthant of $\mathbb{R}^{p}$, that is,

$$
Q=\left\{x \in \mathbb{R}^{p}: x_{i} \geq 0, \forall i=1 \cdots p\right\}
$$


We are interested in the tail distribution of the exit time

$$
\tau_{K}=\inf \left\{n \geq 1: S_{n} \notin K\right\}
$$

of the random walk from the cartesian product

$$
K=Q \times \mathbb{R}^{q},
$$

in the favorable case where $m \in Q \times\{0\}^{q}$, but with the additional constraint that some zero-mean coordinates of the walk stay in a bounded domain. In what follows, we assume the random walk is well-oriented (with respect to $K$ ), that is, the probability distribution satisfies the following condition:

$\left(\mathrm{H}^{\prime}\right)$ The support of $\mu$ is not included in any half-space $u^{-}=\left\{x \in \mathbb{R}^{d}:\langle x\right.$, $u\rangle \leq 0\}$ with $u \in Q \times\{0\}^{q} \backslash\{0\}$.

Let $J$ be the set of indices $j$ such that $m_{j}=0$. Denote by $\mathbb{R}^{(J)}$ the subspace of $J$-coordinates, that is

$$
\mathbb{R}^{(J)}=\left\{x \in \mathbb{R}^{d}: x_{i}=0, \forall i \notin J\right\},
$$

and let $x^{(J)}$ be the projection of $x \in \mathbb{R}^{d}$ on $\mathbb{R}^{(J)}$. Let also

$$
\|x\|_{J}=\left\|x^{(J)}\right\|=\left(\sum_{i \in J}\left|x_{i}\right|^{2}\right)^{1 / 2}
$$

be the norm of the projection of $x$ on the subspace of $J$-coordinates.

We shall prove in this setting the following result that extends Theorem 13 of Garbit and Raschel (2016).

Proposition 1. Assume $\mu$ satisfies $\left(\mathrm{H}^{\prime}\right)$, and that $m \in Q \times\{0\}^{q}$. Let $J$ be the set of indices $j$ such that $m_{j}=0$. There exists $\delta \geq 0$ such that

$$
\lim _{R \rightarrow \infty} \liminf _{n \rightarrow \infty} \mathbb{P}_{\mu}^{x}\left(\tau_{K}>n, \max _{k \leq n}\left\|S_{k}-x\right\|_{J} \leq R\right)^{1 / n}=1
$$

for all $x \in K_{\delta}=Q_{\delta} \times \mathbb{R}^{q}$.

Proposition 1 will follow from the two lemmas below. Roughly speaking, the first one will enable us to push the random walk as far as we want from the boundary of the cone $K$ (with a positive probability):

Lemma 6. Under the hypotheses of Proposition 1 , there exist $\gamma>0, b \geq 1$ and $\delta, R>0$ such that

$$
\mathbb{P}_{\mu}^{x}\left(\tau_{K}>b \ell, S_{b \ell} \in K_{\ell}, \max _{k \leq b \ell}\left\|S_{k}-x\right\|_{J} \leq R\right) \geq \gamma^{\ell},
$$

for all $\ell \geq 1$ and $x \in K_{\delta}$. 
Now, as soon as the random walk has reached a point $y$ at a distance $\geq R$ from the boundary, it suffices that the walk stays in $B(y, R)$ so as to be sure that it will not leave the cone. This simple observation will enable us to derive the theorem from the second proposition.

Lemma 7. Assume $\left(\widetilde{S}_{n}\right)$ is square integrable random walk with mean $m=0$ and any variance-covariance matrix. Then

$$
\lim _{R \rightarrow \infty} \liminf _{n \rightarrow \infty} \mathbb{P}_{\mu}^{0}\left(\max _{k \leq n}\left\|\widetilde{S}_{k}\right\| \leq R\right)^{1 / n}=1
$$

The proofs of Lemmas 6 and 7 are deferred to Section 3.2 and Section 3.3, respectively. First of all, let us explain precisely how the combination of those two propositions leads to Proposition 1.

\subsection{Proof of Proposition 1}

Lemma 6 ensures the existence of $\gamma>0, b \geq 1$ and $\delta, R_{0}>0$ such that

$$
\mathbb{P}_{\mu}^{x}\left(\tau_{K}>b \ell, S_{b \ell} \in K_{\ell}, \max _{k \leq b \ell}\left\|S_{k}-x\right\|_{J} \leq R_{0}\right) \geq \gamma^{\ell},
$$

for all $\ell \geq 1$ and $x \in K_{\delta}$.

Let $\varepsilon>0$ be given. Applying Lemma 7 to the centered random walk $\widetilde{S}_{n}=$ $S_{n}-n m$, we obtain the existence of a number $R \geq R_{0}$ such that

$$
\mathbb{P}_{\mu}^{0}\left(\max _{k \leq n}\left\|\widetilde{S}_{k}\right\| \leq R-R_{0}\right) \geq(1-\varepsilon)^{n},
$$

for all $n$ large enough. Now fix $\ell \geq R-R_{0}$ and suppose that $y \in K_{\ell} \cap \overline{B_{J}\left(x, R_{0}\right)}$. If

$$
\max _{k \leq n}\left\|\widetilde{S}_{k}-y\right\| \leq R-R_{0}(\leq \ell)
$$

then:

1. Clearly, $\widetilde{S}_{k}$ belongs to $K$ for all $k \leq n$. Since $S_{k}=\widetilde{S}_{k}+k m$ and $m$ belongs to $K$, the same is true for $S_{k}$, thus $\tau_{K}>n$.

2. For all $k \leq n$, we have

$$
\left\|S_{k}-x\right\|_{J}=\left\|\widetilde{S}_{k}-x\right\|_{J} \leq\left\|\widetilde{S}_{k}-y\right\|+\|y-x\|_{J} \leq R .
$$

Therefore, if we consider only trajectories such that $S_{b \ell} \in K_{\ell}$ and $\left\|S_{b \ell}-x\right\|_{J} \leq$ $R_{0}$, and then use the Markov property at time $b \ell$, we obtain the lower bound

$$
\begin{aligned}
& \mathbb{P}_{\mu}^{x}\left(\tau_{K}>n, \max _{k \leq n}\left\|S_{k}-x\right\|_{J} \leq R\right) \\
& \quad \geq \gamma^{\ell} \times \inf _{y} \mathbb{P}_{\mu}^{y}\left(\tau_{K}>n-b \ell, \max _{k \leq n-b \ell}\left\|S_{k}-x\right\|_{J} \leq R\right)
\end{aligned}
$$




$$
\begin{aligned}
& \geq \gamma^{\ell} \times \inf _{y} \mathbb{P}_{\mu}^{y}\left(\max _{k \leq n-b \ell}\left\|\widetilde{S}_{k}-y\right\| \leq R-R_{0}\right) \\
& \geq \gamma^{\ell} \times(1-\varepsilon)^{n-b \ell}
\end{aligned}
$$

where the infimum is taken over all $y \in K_{\ell} \cap \overline{B_{J}\left(x, R_{0}\right)}$. Consequently

$$
\liminf _{n \rightarrow \infty} \mathbb{P}_{\mu}^{x}\left(\tau_{K}>n, \max _{k \leq n}\left\|S_{k}-x\right\|_{J} \leq R\right)^{1 / n} \geq 1-\varepsilon,
$$

and the theorem is proved.

\subsection{Pushing the walk deep inside the cone}

This section is devoted to the proof of Lemma 6. In what follows, the distribution $\mu$ of the random walk increments is assumed to satisfy assumption $\left(\mathrm{H}^{\prime}\right)$. Let $m$ be the expectation of $\mu$ and

$$
F=(\operatorname{ker} \Gamma)^{\perp},
$$

where $\Gamma$ is the variance-covariance matrix of $\mu$. It is well-known that the smallest affine subspace of $\mathbb{R}^{d}$ with full $\mu$-probability is

$$
m+F \text {. }
$$

Therefore, assumption $\left(\mathrm{H}^{\prime}\right)$ ensures that there exists no $u \in Q \times\{0\}^{q} \backslash\{0\}$ such that $m+F \subset u^{-}$.

We define the smoothed support $\mathcal{G}$ of the random walk as

$$
\mathcal{G}=\mathbb{R}_{+} m+F
$$

and notice that, if started at any point in $\mathcal{G}$, the random walk stays in $\mathcal{G}$ forever.

In addition, we assume that

$$
m \in Q \times\{0\}^{q},
$$

and define the set

$$
J=\left\{j \in \llbracket 1, d \rrbracket: m_{j}=0\right\} \supset \llbracket p+1, q \rrbracket .
$$

Finally, let

$$
B_{J}(0, R)=\left\{x \in \mathbb{R}^{d}:\|x\|_{J}<R\right\},
$$

where $\|\cdot\|_{J}$ is defined as in (14). 
3.2.1 Some geometry. We collect here two technical lemmas related to the geometry of the problem. The first one asserts that the affine support of $\mu$ meets the interior of the cone $K=Q \times \mathbb{R}^{q}$. This is crucial since otherwise we couldn't expect the walk to go deep inside the cone.

Lemma 8. Assume $\left(\mathrm{H}^{\prime}\right)$ is satisfied and $m \in Q \times\{0\}^{q}$. Then

$$
(m+F) \cap K^{o} \neq \varnothing .
$$

Moreover, for all $x \in K \cap \mathcal{G}$,

$$
(x+m+F) \cap K^{o} \cap B_{J}(0,1) \neq \varnothing .
$$

Proof. We assume $(m+F) \cap K^{o}=\varnothing$, and infer the existence of some $u \in Q \times$ $\{0\}^{q}$ such that $(m+F) \subset u^{\perp}$, thus contradicting the assumption $\left(\mathrm{H}^{\prime}\right)$.

Let us first consider the case where $F=H$ is a hyperplane. Then there exists $u \neq 0$ such that $H=u^{\perp}$. We shall prove that $m \in u^{\perp}$. Suppose on the contrary that $\langle m, u\rangle \neq 0$, then, possibly changing $u$ to $-u$, we can assume that $\langle m, u\rangle>0$. Now, using the homogeneity of $H$ and $K^{o}$, we see that $K^{o}$ does not intersect with

$$
\bigcup_{\lambda>0}(\lambda m+H)=\bigcup_{\lambda>0}\left(\lambda u+u^{\perp}\right)=\{x:\langle x, u\rangle>0\} .
$$

Therefore, $K^{o}$ is included in $u^{-}$. But since $m \in K=\overline{\left(K^{o}\right)}$ (this equality holds for any convex set with non-empty interior), we obtain that $\langle m, u\rangle \leq 0$, which contradicts our hypothesis. Hence, $m$ belongs to $u^{\perp}$ and $m+H=u^{\perp}$. Finally, the non-intersecting hypothesis rewrites $u^{\perp} \cap K^{o}=\varnothing$ and is easily seen to be equivalent to $u \in \pm Q \times\{0\}^{q}$.

We now turn to the general case where $F$ is any linear subspace. Since $m+F$ and $K^{o}$ are two disjoints convex sets, it follows from the Hyperplane separation theorem that there exists an affine hyperplane $H_{m}$ that separates $m+F$ and $K^{o}$. But, since $m$ belongs to both $m+F$ and $K=\overline{K^{o}}$, it must belong to $H_{m}$, and therefore $H_{m}=m+H$, where $H$ is a linear hyperplane. Now, $F$ being a linear subspace, it can't be on one side of $H$ unless it is contained in $H$. Therefore, we obtain that $m+F \subset m+H$ and $(m+H) \cap K^{o}=\varnothing$, and equation (15) follows by applying the first part of the proof to $m+H$.

Let us now show that (15) implies (16). Since $(m+F) \cap K^{o}$ is non-empty, there is some $f_{0} \in F$ such that $m+f_{0} \in K^{o}$. Therefore,

$$
m+\alpha f_{0} \in K^{o}
$$

for all $\alpha \in(0,1]$ (since $m \in K$ and $K$ is convex). Fix such an $\alpha$ so small that $\left\|\alpha f_{0}\right\|_{J}<1$. For $x \in K \cap \mathcal{G}$, write $x=\lambda_{1} m+f_{1}$ with $\lambda_{1} \geq 0$ and $f_{1}$ in $F$, and set $f=\alpha f_{0}-f_{1}$. Then,

$$
x+m+f=\lambda_{1} m+\left(m+\alpha f_{0}\right) \in K^{o} .
$$


(Since $\lambda_{1} m \in K, m+\alpha f_{0} \in K^{o}$ and $K+K^{o} \subset K^{o}$.) In addition,

$$
\|x+m+f\|_{J}=\left\|\alpha f_{0}\right\|_{J}<1,
$$

thus proving that $x+m+f \in K^{o} \cap B_{J}(0,1)$.

The second lemma is a technical tool. For any $x, y \in \mathbb{R}^{d}$, we write $y \leq x$ iff $x-y \in \mathbb{R}_{+}^{d}$.

Lemma 9. Let $\left(x_{n}\right)$ be a sequence in $K \cap \overline{B_{J}(0,1)} \cap \mathcal{G}$. There exists a bounded sequence $\left(y_{n}\right)$ in $K \cap \overline{B_{J}(0,1)} \cap \mathcal{G}$, such that

$$
y_{n} \leq x_{n} \quad \text { and } \quad y_{n}^{(J)}=x_{n}^{(J)} \quad \text { for all } n .
$$

Proof. Since $\mathcal{G}=\mathbb{R}_{+} m+F$, where $F$ is a linear subspace of $\mathbb{R}^{d}$, there exist $L \in$ $M_{d}(\mathbb{R})$ and a linear form $\phi: \mathbb{R}^{d} \rightarrow \mathbb{R}$, such that

$$
\mathcal{G}=\left\{x \in \mathbb{R}^{d}: L(x)=0, \phi(x) \geq 0\right\} .
$$

Recall that

$$
\mathbb{R}^{(J)}=\left\{x \in \mathbb{R}^{d}: x_{i}=0, \forall i \notin J\right\} .
$$

Let $\mathbb{R}^{(I)}$ be its orthogonal complement, that is,

$$
\mathbb{R}^{(I)}=\left\{x \in \mathbb{R}^{d}: x_{i}=0, \forall i \in J\right\},
$$

and write $x=x^{(I)}+x^{(J)}$ the orthogonal decomposition with respect to $\mathbb{R}^{(I)} \oplus$ $\mathbb{R}^{(J)}$. Let $x_{n}=x_{n}^{(I)}+x_{n}^{(J)}$ be an element of $K \cap \overline{B_{J}(0,1)} \cap \mathcal{G}$ and define

$$
P=\left\{z \in \mathbb{R}_{+}^{(I)}: L(z)=-L\left(x_{n}^{(J)}\right), \phi(z) \geq-\phi\left(x_{n}^{(J)}\right)\right\} .
$$

For all $z \in \mathbb{R}_{+}^{(I)}$, notice that $z \in P$ iff $z+x_{n}^{(J)} \in \mathcal{G}$. Therefore, $x_{n}^{(I)} \in P$. It follows from Corollary 1 in Appendix that there exists $y_{n}^{(I)} \in P$ such that $y_{n}^{(I)} \leq x_{n}^{(I)}$ and

$$
\left\|y_{n}^{(I)}\right\| \leq M\left(\left\|L\left(x_{n}^{(J)}\right)\right\|+\left|\phi\left(x_{n}^{(J)}\right)\right|\right),
$$

where $M=M(L, \phi)$ only depends on $L$ and $\phi$ (and not on $x_{n}$ ). Setting $y_{n}=$ $y_{n}^{(I)}+x_{n}^{(J)}$ thus gives a bounded sequence in $K \cap \overline{B_{J}(0,1)} \cap \mathcal{G}$ that satisfies the conditions of the lemma.

3.2.2 Proof of Lemma 6. We begin with a lemma that asserts the existence of a time $b$ and a radius $R_{0}$ such that the random walk started at $x \in K \cap \mathcal{G}$ with $\|x\|_{J} \leq R_{0}$ will be at a distance $\geq 1$ from the boundary of $K$ at time $b$ and still located in $\overline{B_{J}\left(0, R_{0}\right)}$ with a probability that is bounded from below by some positive constant, uniformly in $x$. 
Lemma 10. There exist $b \geq 1$ and $R_{0}>0$ such that

$$
\underset{x \in K \cap \frac{\inf }{B_{J}\left(0, R_{0}\right)} \cap \mathcal{G}}{ } \mathbb{P}_{\mu}^{x}\left(S_{b} \in K_{1},\left\|S_{b}\right\|_{J} \leq R_{0}\right)>0 .
$$

Proof. Clearly, the lemma will follow from the existence of an integer $n \geq 1$ such that

$$
\inf _{x \in K \cap B_{J}(0,1) \cap \mathcal{G}} \mathbb{P}_{\mu}^{x \sqrt{n}}\left(S_{n} \in K_{1},\left\|S_{n}\right\|_{J} \leq \sqrt{n}\right)>0 .
$$

So, let us assume that this assertion is false. Then, we can find a sequence of points $x_{n} \in K \cap \overline{B_{J}(0,1)} \cap \mathcal{G}$ such that

$$
p_{n}:=\mathbb{P}_{\mu}^{x_{n} \sqrt{n}}\left(S_{n} \in K_{1},\left\|S_{n}\right\|_{J} \leq \sqrt{n}\right) \rightarrow 0 .
$$

Thanks to Lemma 9, we can assume that $\left(x_{n}\right)$ is bounded, because for any sequence $\left(y_{n}\right)$ with the same properties as in this lemma, the probability $p_{n}$ where $x_{n}$ is replaced by $y_{n}$ is smaller than $p_{n}$, by inclusion of events and convexity of the cone. Furthermore, by extracting a subsequence, it can be assumed without loss of generality that $\left(x_{n}\right)$ converges to some element $x$ of the closed set $K \cap$ $\overline{B_{J}(0,1)} \cap \mathcal{G}$.

Now, let $\widetilde{S}_{n}=S_{n}-n m$ denote the centered random walk associated with $S_{n}$. Since $\|\cdot\|_{J}$ is left invariant by a translation by $m$, the probability $p_{n}$ can be written as

$$
p_{n}=\mathbb{P}_{\mu}^{0}\left(x_{n} \sqrt{n}+\widetilde{S}_{n} \in K_{1}-n m,\left\|x_{n} \sqrt{n}+\widetilde{S}_{n}\right\|_{J} \leq \sqrt{n}\right) .
$$

Let $\eta>0$ be fixed. For all $n \geq 1 / \eta$, holds the inclusion

$$
K_{\eta}-m \subset\left(K_{1}-n m\right) / \sqrt{n} \text {. }
$$

Therefore, the probability $p_{n}$ is bounded from below by

$$
\mathbb{P}_{\mu}^{0}\left(x_{n}+\widetilde{S}_{n} / \sqrt{n} \in K_{\eta}-m,\left\|x_{n}+\widetilde{S}_{n} / \sqrt{n}\right\|_{J} \leq 1\right) .
$$

Since $x_{n}+\widetilde{S}_{n} / \sqrt{n}$ converges in distribution to $x+X$, where $X$ denotes a random variable with $\mathcal{N}(0, \Gamma)$ Gaussian distribution, we can use the Portmanteau theorem and let then $\eta \downarrow 0$ to get the lower bound

$$
\liminf _{n \rightarrow \infty} p_{n} \geq \mathbb{P}\left(x+X \in\left(K^{o}-m\right) \cap B_{J}(0,1)\right) .
$$

The random variable $x+X$ admits a positive density with respect to Lebesgue measure on the affine space $x+F$ where $F=(\operatorname{ker} \Gamma)^{\perp}$, and

$$
O:=(x+F) \cap\left(K^{o}-m\right) \cap B_{J}(0,1)
$$

is an open subset of $x+F$. Thus it suffices to prove that $O$ is non-empty to obtain a contradiction. But this is precisely what Lemma 8 asserts: Indeed, since $x$ belongs to $K \cap \mathcal{G}$, we obtain that

$$
(x+m+F) \cap K^{o} \cap B_{J}(0,1) \neq \varnothing .
$$


Hence, subtracting $m$ on both sides (recall that $\|\cdot\|_{J}$ is left invariant by a translation by $m$ ) gives

$$
O \neq \varnothing
$$

This implies

$$
\mathbb{P}(x+X \in O)>0,
$$

thus contradicting our assumption that $\liminf p_{n}=0$. Therefore, the lemma is proven.

By Lemma 10, there exist $b \geq 1$ and $R_{0}>0$ such that, for $\gamma>0$ small enough,

$$
\mathbb{P}_{\mu}^{x}\left(S_{b} \in K_{1},\left\|S_{b}\right\|_{J} \leq R_{0}\right) \geq 2 \gamma,
$$

for all $x \in K \cap \overline{B_{J}\left(0, R_{0}\right)} \cap \mathcal{G}$. Let us choose $\delta>0$ and $R \geq R_{0}$ such that

$$
\mathbb{P}_{\mu}^{0}\left(\tau_{K_{-\delta}}>b, \max _{k \leq b}\left\|S_{k}\right\|_{J} \leq R-R_{0}\right) \geq 1-\gamma .
$$

Then, by inclusion of events, we also have

$$
\mathbb{P}_{\mu}^{x}\left(\tau_{K_{-\delta}}>b, \max _{k \leq b}\left\|S_{k}\right\|_{J} \leq R\right) \geq 1-\gamma,
$$

for all $x \in K \cap \overline{B_{J}\left(0, R_{0}\right)} \cap \mathcal{G}$. Indeed, this follows from the relation $K+K_{-\delta} \subset$ $K_{-\delta}$ and the triangle inequality for $\|\cdot\|_{J}$. Now, combining (17) and (18), we obtain that

$$
\mathbb{P}_{\mu}^{x}\left(\tau_{K_{-\delta}}>b, S_{b} \in K_{1}, \max _{k \leq b}\left\|S_{k}\right\|_{J} \leq R,\left\|S_{b}\right\|_{J} \leq R_{0}\right) \geq \gamma
$$

for all $x \in K \cap \overline{B_{J}\left(0, R_{0}\right)} \cap \mathcal{G}$. Set

$$
p_{\ell}(x)=\mathbb{P}_{\mu}^{x}\left(\tau_{K_{-\delta}}>b \ell, S_{b \ell} \in K_{\ell}, \max _{k \leq b \ell}\left\|S_{k}\right\|_{J} \leq R\right) .
$$

Notice that $K_{1}+K_{\ell} \subset K_{\ell+1}$. Hence, if we consider only trajectories such that $S_{b} \in K_{1}$ and $\left\|S_{b}\right\|_{J} \leq R_{0}$, and then use the Markov property at time $b$, we get the lower bound

$$
p_{\ell+1}(x) \geq \gamma \times \underset{y \in K \cap \frac{\inf }{B_{J}\left(0, R_{0}\right)} \cap \mathcal{G}}{ } p_{\ell}(y),
$$

for all $x \in K \cap \overline{B_{J}\left(0, R_{0}\right)} \cap \mathcal{G}$. This proves that $p_{\ell}(0) \geq \gamma^{\ell}$ for all $\ell \geq 1$, and Lemma 6 follows by inclusion of events since $K_{\delta}+K_{-\delta} \subset K$ and $K_{\delta}+K_{\ell} \subset K_{\ell}$.

\subsection{On the exit time from a ball}

This section is devoted to the proof of Lemma 7. In what follows, the abbreviation "f.s." stands for "for some". 
3.3.1 Preliminary estimate for Brownian motion. Let $\left(B_{t}\right)$ denotes a truly $d$ dimensional Brownian motion, that is, the image of a standard $d$-dimensional Brownian motion under an invertible linear transformation.

Lemma 11. For every $\varepsilon>0$, there exist $\delta>0$ and $0<\alpha<1$ such that

$$
\mathbb{P}^{x}\left(\left\|B_{t}\right\|<1-\delta \text { f.s. } t \in[\alpha, 1]\right) \geq 1-\varepsilon
$$

for all $x \in \overline{B(0,1)}$.

Proof. Suppose on the contrary that there is some $\varepsilon_{0}>0$ for which we can pick $\alpha_{n} \downarrow 0, \delta_{n} \downarrow 0$ and $x_{n} \in \overline{B(0,1)}$ such that

$$
p_{n}:=\mathbb{P}^{x_{n}}\left(\left\|B_{t}\right\|<1-\delta_{n} \text { f.s. } t \in\left[\alpha_{n}, 1\right]\right)<1-\varepsilon_{0}
$$

for all $n$. By compactness, it can also be assumed that $\left\|x_{n}-x\right\| \rightarrow 0$ for some $x \in \overline{B(0,1)}$. Now, for any $\eta>0$, we have

$$
p_{n} \geq \mathbb{P}^{x}\left(\left\|B_{t}\right\|<1-\eta \text { f.s. } t \in\left[\alpha_{n}, 1\right]\right)
$$

as soon as $\left\|x_{n}-x\right\|+\delta_{n} \leq \eta$. Hence, taking the limit on both sides and letting $\eta \downarrow 0$ gives

$$
\liminf _{n \rightarrow \infty} p_{n} \geq \mathbb{P}^{x}\left(\left\|B_{t}\right\|<1 \text { f.s. } t \in(0,1]\right) .
$$

But it follows from the classical cone condition (as found in Port and Stone (1978), Proposition 3.3, for example) applied to the ball $B(0,1)$ that $x$ is regular for $B(0,1)$, that is, $B_{t}$ immediately visits $B(0,1)$ with full probability. Therefore, the last inequality reads

$$
\liminf _{n \rightarrow \infty} p_{n} \geq 1
$$

and contradicts our assumption.

3.3.2 Application to random walks. In this subsection, $\left(S_{n}\right) \in \mathbb{R}^{d}$ is a square integrable random walk with increments distribution $\mu$, mean $m=0$ and any covariance matrix $\Gamma$.

The proof of Lemma 7 is based on the following basic idea. Given $\varepsilon>0$, find $R>0$ and a time $n_{0} \geq 1$ such that the random walk started at 0 returns to 0 at time $n_{0}$ without leaving the ball $B(0, R)$ with probability $\geq 1-\varepsilon$. If this can be done, then the result follows by concatenation (i.e., Markov property). But this is asking for a property stronger than recurrence, thus we can not hope for such a simple argument. Instead of a return to 0 , we can ask for a return in some ball $B\left(0, R_{0}\right)$, with $R_{0} \leq R$. But then, in view of using concatenation, we need the previous probability to be greater than $1-\varepsilon$ uniformly for any starting point in the same ball $B\left(0, R_{0}\right)$. Lemma 12 below provides a result in this spirit that is sufficient for our purpose. 
Lemma 12. Suppose (here only) that the covariance matrix $\Gamma$ is non-degenerate. Then, for every $\varepsilon>0$, there exist $0<R_{0}<R$ and $1 \leq \ell_{0} \leq n_{0}$ such that

$$
\mathbb{P}_{\mu}^{x}\left(\max _{k \leq n_{0}}\left\|S_{n}\right\| \leq R \text { and }\left\|S_{k}\right\| \leq R_{0} \text { f.s. } k \in \llbracket \ell_{0}, n_{0} \rrbracket\right) \geq 1-\varepsilon
$$

for all $x \in \overline{B\left(0, R_{0}\right)}$.

Proof. Let $\varepsilon>0$ be given and fix $\delta>0$ and $0<\alpha<1$ so that the conclusion of Lemma 11 holds. Also, fix a parameter $\beta \in(0, \alpha)$.

Let $\left(x_{n}\right)$ be a sequence in $\overline{B(0,1)}$ that converges to some $x \in \overline{B(0,1)}$, and set

$$
p_{n}=\mathbb{P}_{\mu}^{x_{n} \sqrt{n}}\left(\left\|S_{k}\right\| \leq \sqrt{n} \text { f.s. } k \in[\beta n, n]\right) .
$$

We shall prove that

$$
\liminf _{n \rightarrow \infty} p_{n} \geq 1-\varepsilon .
$$

To do this, we consider the process with continuous path $\left(Z_{n}(t), t \in[0,1]\right)$ defined by

$$
Z_{n}(t)=\frac{S_{[n t]}}{\sqrt{n}}+(n t-[n t]) \frac{\xi_{[n t]+1}}{\sqrt{n}},
$$

where $\xi_{k}=S_{k}-S_{k-1}$ and $[a]$ denotes the integer part of $a$. The probability $p_{n}$ can then be written in terms of the process $Z_{n}$ as $\mathbb{P}_{\mu}^{0}\left(A_{n}\right)$, where

$$
A_{n}=\left\{\left\|x_{n}+Z_{n}(t)\right\| \leq 1 \text { f.s. } t=k / n \in[\beta, 1]\right\} .
$$

We wish to use the Functional central limit theorem Billingsley (1968), Theorem 10.1, together with the Portmanteau theorem Billingsley (1968), Theorem 2.1, in order to obtain a lower bound for the probability of this event, but the condition that $t$ be rational $(t=k / n)$ can not be handled directly, and must therefore be relaxed. To this end, we define

$$
\widetilde{A}_{n}=\left\{\left\|x+Z_{n}(t)\right\|<1-\delta \text { f.s. } t \in[\alpha, 1]\right\}
$$

and

$$
B_{n}=\left\{\max _{k=0, \ldots, n}\left\|\xi_{k}\right\|>(\delta / 2) \sqrt{n}\right\} .
$$

Since $\left\|Z_{n}(t)-Z_{n}(k / n)\right\| \leq\left\|\xi_{k+1}\right\| / \sqrt{n}$ for $k / n \leq t<(k+1) / n$, we have the inclusion of events

$$
\widetilde{A}_{n} \cap \overline{B_{n}} \subset A_{n}
$$

as soon as $\left\|x_{n}-x\right\| \leq \delta / 2$ and $\alpha-\beta>1 / n$. Thus, for all sufficiently large $n$, we have

$$
p_{n} \geq \mathbb{P}_{\mu}^{0}\left(\widetilde{A}_{n}\right)-\mathbb{P}_{\mu}^{0}\left(B_{n}\right)
$$


Furthermore, it is a basic result in probability theory that $\mathbb{P}\left(B_{n}\right) \rightarrow 0$ for any i.i.d. sequence of zero-mean square integrable random variables $\left(\xi_{k}\right)$. Hence, we are left to prove that

$$
\liminf _{n \rightarrow \infty} \mathbb{P}_{\mu}^{0}\left(\widetilde{A}_{n}\right) \geq 1-\varepsilon .
$$

Now, since $\Gamma$ is non-degenerate, the Functional central limit theorem asserts that $Z_{n}$ converges in distribution to a true $d$-dimensional Brownian motion (with covariance matrix $\Gamma$ ) on the space of continuous functions $w:[0,1] \rightarrow \mathbb{R}^{d}$ equipped with the topology of uniform convergence. Since the set of continuous functions $w:[0,1] \rightarrow \mathbb{R}^{d}$ such that $\|w(t)\|<1-\delta$ for some $t \in[\alpha, 1]$ is open with respect to the topology of uniform convergence, it follows from the Portmanteau theorem that

$$
\liminf _{n \rightarrow \infty} \mathbb{P}_{\mu}^{0}\left(\widetilde{A}_{n}\right) \geq \mathbb{P}^{0}\left(\left\|x+B_{t}\right\|<1-\delta \text { f.s. } t \in[\alpha, 1]\right) \geq 1-\varepsilon,
$$

where the lower bound $1-\varepsilon$ comes from our choice of $\delta$ and $\alpha$. This proves our first claim that $\liminf _{n} p_{n} \geq 1-\varepsilon$. By a standard compactness argument, this immediately implies that

$$
\inf _{x \in \overline{B(0,1)}} \mathbb{P}_{\mu}^{x \sqrt{n}}\left(\left\|S_{k}\right\| \leq \sqrt{n} \text { f.s. } k \in[\beta n, n]\right) \geq 1-2 \varepsilon
$$

for all sufficiently large $n$. Fix such $n_{0}>1 / \beta$ and set $\ell_{0}=\left[\beta n_{0}\right]$ and $R_{0}=\sqrt{n_{0}}$. Then we have $0<\ell_{0}<n_{0}$, and the last inequality can be rewritten as

$$
\inf _{x \in B\left(0, R_{0}\right)} \mathbb{P}_{\mu}^{x}\left(\left\|S_{k}\right\| \leq R_{0} \text { f.s. } k \in \llbracket \ell_{0}, n_{0} \rrbracket\right) \geq 1-2 \varepsilon .
$$

In order to complete the proof, it suffices to notice that, for all $x \in \overline{B(0,1)}$ and $R \geq R_{0}$, we have

$$
\mathbb{P}_{\mu}^{x}\left(\max _{k \leq n_{0}}\left\|S_{k}\right\| \leq R\right) \geq \mathbb{P}_{\mu}^{0}\left(\max _{k \leq n_{0}}\left\|S_{k}\right\| \leq R-R_{0}\right) .
$$

Since the last probability goes to 1 as $R \rightarrow \infty$, it is bounded from below by $1-\varepsilon$ for all sufficiently large $R$, and we conclude that for such a choice of $R$, we have

$$
\mathbb{P}_{\mu}^{x}\left(\max _{k \leq n_{0}}\left\|S_{k}\right\| \leq R \text { and }\left\|S_{k}\right\| \leq R_{0} \text { f.s. } k \in \llbracket \ell_{0}, n_{0} \rrbracket\right) \geq 1-3 \varepsilon
$$

for all $x \in \overline{B\left(0, R_{0}\right)}$.

3.3.3 Proof of Lemma 7. First of all, we notice that the variance-covariance matrix $\Gamma$ can be assumed to be non-degenerate. Otherwise, the random walk lives on $(\operatorname{ker} \Gamma)^{\perp}$ where it has a non-degenerate variance-covariance matrix. Since the projection of a $d$-dimensional ball is still a ball in $(\operatorname{ker} \Gamma)^{\perp}$, the result will follow by application of the non-degenerate case to the projected random walk. 
As explained earlier, the idea is now to concatenate the "high-probability path" given by Lemma 12. Let $\varepsilon>0$ be given. Thanks to Lemma 12, we can find $\delta>0$, $0<R_{0}<R$ and $1 \leq \ell_{0} \leq n_{0}$ such that

$$
\inf _{x \in \overline{B\left(0, R_{0}\right)}} \mathbb{P}_{\mu}^{x}\left(\max _{k \leq n_{0}}\left\|S_{n}\right\| \leq R, H \leq n_{0}\right) \geq 1-\varepsilon,
$$

where $H$ denotes the first hitting time of the ball $\overline{B\left(0, R_{0}\right)}$ after time $\ell_{0}$.

For $x \in \overline{B\left(0, R_{0}\right)}$ and $n \geq n_{0}$, we use the strong Markov property at time $H$ to get

$$
\begin{aligned}
& \mathbb{P}_{\mu}^{x}\left(\max _{k \leq n}\left\|S_{k}\right\| \leq R\right) \\
& \quad \geq \mathbb{P}_{\mu}^{x}\left(\max _{k \leq n}\left\|S_{k}\right\| \leq R, H \leq n_{0}\right) \\
& \quad \geq \mathbb{E}_{\mu}^{x}\left(\max _{k \leq H}\left\|S_{k}\right\| \leq R, H \leq n_{0}, \mathbb{P}_{\mu}^{S_{H}}\left(\max _{k \leq n-j}\left\|S_{k}\right\| \leq R\right)_{\mid j=H}\right) \\
& \quad \geq \mathbb{P}_{\mu}^{x}\left(\max _{k \leq H}\left\|S_{k}\right\| \leq R, H \leq n_{0}\right) \times \underset{y \in \overline{B\left(0, R_{0}\right)}}{\inf } \mathbb{P}_{\mu}^{y}\left(\max _{k \leq n-\ell_{0}}\left\|S_{k}\right\| \leq R\right) .
\end{aligned}
$$

Thus,

$$
R(n):=\inf _{x \in B\left(0, R_{0}\right)} \mathbb{P}_{\mu}^{x}\left(\max _{k \leq n}\left\|S_{k}\right\| \leq R\right)
$$

satisfies the inequality

$$
R(n) \geq(1-\varepsilon) R\left(n-\ell_{0}\right)
$$

for all $n \geq n_{0}$. Since $R(n)$ is clearly decreasing, for $n_{0}+k \ell_{0} \leq n<n_{0}+(k+1) \ell_{0}$, we obtain

$$
R(n) \geq R\left(n_{0}+(k+1) \ell_{0}\right) \geq(1-\varepsilon)^{k+1} R\left(n_{0}\right) \geq(1-\varepsilon)^{n+2} .
$$

Hence,

$$
\liminf _{n \rightarrow \infty} R(n)^{1 / n} \geq 1-\varepsilon
$$

and this proves the lemma.

\section{Appendix: Minimal points of a polyhedron}

This independent and (nearly) self-contained section provides the material we need for the proof of Lemma 9. The notion of minimality introduced here and the related results are certainly not new, but we were not able to find any reference for them. Nevertheless, the proofs will only be sketched since the arguments are very similar to the standard ideas of linear programming theory, as can be found in the book (Ciarlet (1982)) for example. 
Let $1 \leq m \leq n$. We consider here the polyhedron

$$
P=\left\{x \in \mathbb{R}_{+}^{n}: \sum_{i=1}^{n} x_{i} C_{i}=b\right\},
$$

where $C_{i}, b$ are any vectors of $\mathbb{R}^{m}$, and $x_{i}$ denote the coordinates of $x$ in the standard basis.

If $P$ is not empty (a condition that we assume from now on), it is a closed convex set. Its extremal points are called the vertices of $P$. Note that 0 is a vertex iff $b=0$. There exist a simple and well known characterization of vertices. To $x \in P$, let us associate the subset $I(x)$ of indices $i$ such that $x_{i}>0$. Then $x \neq 0$ is a vertex of $P$ iff the vectors $\left\{C_{i}, i \in I(x)\right\}$ are linearly independent (see Ciarlet (1982), Théorème 10.3-1). It follows easily that there is only a finite number of vertices. The fact that there always exist (at least) a vertex is proved in Ciarlet (1982), Théorème 10.3-3, for example, and will follow as a by-product of our analysis.

For $x, y \in \mathbb{R}^{n}$, we write $y \leq x$ iff $x-y \in \mathbb{R}_{+}^{n}$ and $y<x$ iff $y \leq x$ and $y \neq x$. Hence $x>0$ means that $x \geq 0$ and one of its coordinates (at least) is $>0$. We shall say that $x \in P$ is minimal if $P$ does not contain any $y<x$.

Let us begin with a characterization of minimality.

Lemma 13. An element $x \neq 0$ of $P$ is minimal iff the vectors $\left\{C_{i}, i \in I(x)\right\}$ are positively independent, that is:

$$
\sum_{i \in I(x)} u_{i} C_{i}=0 \quad \text { and } \quad u_{i} \geq 0 \quad \text { for all } i \in I(x) \Rightarrow u_{i}=0 \quad \text { for all } i \in I(x) .
$$

Proof. Let $x$ be an element of $P$ and assume first that $x$ is not minimal. Then there exist $y \in P$ such that $y<x$. Set $u_{i}=x_{i}-y_{i}$ for $i \in I(x)$. Then, the $u_{i}$ 's are $\geq 0$, at least one of them is $>0$, and

$$
\sum_{i \in I(x)} u_{i} C_{i}=0 .
$$

Hence, the vectors $\left\{C_{i}, i \in I(x)\right\}$ are not positively independent.

Conversely, suppose that there exist non-negative numbers $u_{i}, i \in I(x)$, not all zero, such that relation (21) holds. For $i \notin I(x)$, set $u_{i}=0$, and let $u \in \mathbb{R}_{+}^{n}$ denote the vector with coordinates $u_{i}$. Since one of the $u_{i}$ 's is $>0$, we have $y=x-t u<x$ for any $t>0$. Now, if $t>0$ is chosen so small that $y \geq 0$, then $y$ belongs to $P$, hence $x$ is not minimal.

From this lemma, it follows that any vertex of $P$ is minimal. Let us denote by $P_{m}$ the set of minimal points of $P$. The main result of this appendix is the following proposition.

Proposition 2. Assume $P \neq \varnothing$. Then, 
1. For any $x \in P$, there exist $y \in P_{m}$ such that $y \leq x$;

2. Every $y \in P_{m}$ is a convex combination of the vertices of $P$;

3. There exists a number $M$, only depending on the $C_{i}$ 's, such that $P_{m}$ is bounded by $M\|b\|$.

Proof. Suppose that $x \in P$ is not minimal (otherwise there is nothing to prove) and construct a vector $u \in \mathbb{R}_{+}^{n}$ exactly as in the converse part of the previous proof. Now we consider the smallest $t>0$ such that $x-t u$ has a new zero coordinate, that is, we define

$$
t=\min \left\{x_{i} / u_{i}: u_{i}>0\right\}>0
$$

and set $y=x-t u$. The point $y$ belongs to $P$, satisfies the inequality $y<x$ and the strict inclusion relation $I(y) \subsetneq I(x)$. If $y$ is not minimal, we repeat the argument (with $y$, and so on) until a minimal point is reached. The process indeed terminates since the set of positive indices $I(\cdot)$ is finite and strictly decreasing along the process. This proves the first part of the proposition.

The proof of the second part is quite similar. Let $x \in P$ be minimal and suppose it is not a vertex of $P$. Then, the vectors $\left\{C_{i}, i \in I(x)\right\}$ are positively independent but not independent. Therefore, there exist real numbers $u_{i}, i \in I(x)$, not all zero, such that (21) is satisfied and at least one of them is $<0$ and another is $>0$. For $i \notin I(x)$, set $u_{i}=0$, and let $u \in \mathbb{R}^{n}$ denote the vector with coordinates $u_{i}$. Define

$$
t^{+}=\min \left\{-x_{i} / u_{i}: u_{i}<0\right\}>0 \text { and } x^{+}=x+t^{+} u,
$$

and similarly,

$$
t^{-}=\min \left\{x_{i} / u_{i}: u_{i}>0\right\}>0 \text { and } x^{-}=x-t^{-} u .
$$

The two points $x^{+}$and $x^{-}$belong to $P$ and are both minimal because so is $x$ and $I\left(x^{ \pm}\right) \subsetneq I(x)$ by construction. Furthermore, $x$ is clearly a convex combination of $x^{+}$and $x^{-}$. If $x^{+}$and $x^{-}$are both vertices of $P$, then the proof is finished. Else, we repeat the argument until $x$ is written as a convex combination of vertices. The process indeed terminates because of the strict inclusions $I\left(x^{ \pm}\right) \subsetneq I(x)$, as in the first part.

For the proof of the third assertion, we first notice that the Manhattan norm $\|x\|_{1}=\sum_{i=1}^{n}\left|x_{i}\right|$ coincides with the linear form $\sum_{i=1}^{n} x_{i}$ on $\mathbb{R}_{+}^{n}$. Hence, it easily follows from the second assertion of the proposition that

$$
\sup _{x \in P_{m}}\|x\|_{1}=\max \left\{\|v\|_{1}: v \text { vertex of } P\right\} .
$$

Let us denote by $\mathcal{I}$ the family of subsets $I \subset \llbracket 1, n \rrbracket$ such that $\left\{C_{i}, i \in I\right\}$ is a family of linearly independent vectors. If $v$ is a vertex of $P$, then $I=I(v)$ belongs to $\mathcal{I}$ and, because of independence, the numbers $v_{i}, i \in I$, are uniquely determined by the relation

$$
\sum_{i \in I} v_{i} C_{i}=b
$$


From that, it is easily seen that there exists a $n \times m$ matrix $A_{I}$, only depending on the vectors $C_{i}, i \in I$, such that $v=A_{I} b$. Therefore, $\|v\|_{1} \leq\left\|A_{I}\right\|_{1}\|b\|_{1}$ and $P_{m}$ is thus bounded by $M\|b\|_{1}$, where $M=\max _{I \in \mathcal{I}}\left\|A_{I}\right\|_{1}$.

In view of application to the proof of Lemma 9, we need to extend some of the consequences of Proposition 2 to a larger class of polyhedra. So, let $L: \mathbb{R}^{n} \rightarrow \mathbb{R}^{n}$ be a linear mapping and $\phi: \mathbb{R}^{n} \rightarrow \mathbb{R}$ be a linear form. Fix $b \in \mathbb{R}^{n}, c \in \mathbb{R}$, and consider the polyhedron

$$
P=\left\{x \in \mathbb{R}_{+}^{n}: L(x)=b, \phi(x) \geq c\right\} .
$$

Corollary 1. Assume $P \neq \varnothing$. There exists a number $M$, only depending on $L$ and $\phi$, such that, for all $x \in P$, there exists $y \in P$ with $y \leq x$ and $\|y\| \leq M(\|b\|+|c|)$.

Proof. As usual in linear programming problems, we use the fact that $P$ is the projection onto $\mathbb{R}^{n}$ (via $x^{\prime}=\left(x, x_{n+1}\right) \in \mathbb{R}^{n} \times \mathbb{R} \mapsto x \in \mathbb{R}^{n}$ ) of the polyhedra

$$
P^{\prime}=\left\{x^{\prime}=\left(x, x_{n+1}\right) \in \mathbb{R}_{+}^{n+1}: L(x)=b, x_{n+1}=\phi(x)-c\right\} .
$$

Clearly the two constraints that define $P^{\prime}$ can be written as $L^{\prime}\left(x^{\prime}\right)=b^{\prime}$ where $x^{\prime}=\left(x, x_{n+1}\right), b^{\prime}=(b,-c)$ and $L^{\prime} \in M_{n+1}(\mathbb{R})$ only depends on $L$ and $\phi$. Now the result follows easily by applying Proposition 2 to $P^{\prime}$.

\section{Acknowledgments}

The author would like to thank Kilian Raschel and an anonymous referee for their valuable comments.

\section{References}

Billingsley, P. (1968). Convergence of Probability Measures. New York: Wiley. MR0233396

Ciarlet, P. G. (1982). Introduction à L'analyse Numérique Matricielle et à L'optimisation. Paris: Masson. MR0680778

Denisov, D. and Wachtel, W. (2015). Random walks in cones. Ann. Probab. 43, 992-1044. MR3342657

Duraj, J. (2014). Random walks in cones: The case of nonzero drift. Stochastic Process. Appl. 124, 1503-1518. MR3163211

Garbit, R. and Raschel, K. (2016). On the exit time from a cone for random walks with drift. Rev. Mat. Iberoam. 32, 511-532. MR3512425 
Port, S. C. and Stone, C. J. (1978). Brownian Motion and Classical Potential Theory. Probability and Mathematical Statistics. San Diego: Academic Press. MR0492329

Département de Mathématiques

Université d'Angers

LAREMA

UMR CNRS 6093

2 Boulevard Lavoisier

49045 Angers Cedex 1

France

E-mail: rodolphe.garbit@univ-angers.fr 